B. Pyrogallol als Reduktionsmittel.

Ferd. Henrich ${ }^{27}$ ) hat kolloides Quecksilber dadurch erhalten, daß er eine genügend Salpetersäure enthaltende wässerige Lösung von Merkuronitrat in Gegenwart von Natriumazetat mittelst Pyrogallol reduzierte. Er fand, daß man, wenn die Hydrosole einigermaßen haltbar sein sollen, von Lösungen ausgehen muß, die nicht viel konzentrierter sind als 1/1000-n., und daß man die Präparate bald und ausgiebig zu dialysieren hat. Die Systeme sind im durchfallenden Lichte dunkel braungelb, im auffallenden Lichte grauwei $\wp$ und bewahren ihren kolloiden Charakter selten länger als einen halben Monat.

$\mathrm{Zu}$ den Versuchen, welche Herr E. Kuhn $\dagger$ auf unsere Veranlassung angestellt hat, wurde eine $1 / 500-n$. Lösung von Merkuronitrat benutzt und mit dem gleichen Volumen der oben genannten

27) Ferd. Hentich, loc. cit.
Leinsamenschleimlösung verdünnt angewandt. Das System enthielt soviel freie Salpetersäure, daß das Quecksilbersalz in Lösung gehalten wurde.

Verfuhr man nun genau nach den von Ferd. Henrich gegebenen Vorschriften, so gewann man Präparate von kolloidem Quecksilber, das alle die von dem genannten Forscher beschriebenen Eigenschaften besaß, sich aber auch nach sogleich angesetzter und lange Zeit fortgesetzter Dialyse nicht als sehr beständig erwies.

Im Verlaufe der Dialyse -selbst erlitten die Präparate keine sichtliche Veränderung; sie ließen sich auch unzersetzt filtrieren, koagulierten aber bald in irreversibler Form. Da es infolgedessen nicht möglich war, feste in Wasser ohne Rückstand wieder zerteilbare Kolloide zu bereiten, sind Versuche in größerem Mafsstabe nicht angestellt worden.

\title{
Bericht über die Fortschritte in der Gerbereichemie in den Jahren 1913-1915.
}

\author{
Von R. Lauffmann (Freiberg i. Sa.).
}

(Eingegangen am 8. Mai 1916.)

$\mathrm{Zu}$ den bedeutenden Fortschritten, die auch auf dem Gebiete der Gerbereiwissenschaft und -technik in neuerer Zeit $z u$ verzeichnen sind, hat das Wirken des Internationalen Vereins der Lederindustrie-Chemiker außerordentlich viel beigetragen. Wie schon eine Reihe von Jahren vorher, so hatten auch im Jahre 1914 die Mitglieder aller Sektionen des Vereins gehofft, in friedlichem Wettbewerb zum Nutzen der gesamten Lederindustrie weiter $\mathrm{zu}$ arbeiten. Schon waren alle Vorbereitungen für die 12. Hauptversammlung des Vereins, die vom 24 . bis 29. August jenes Jahres in Wien stattfinden sollte, getroffen, als der Krieg ein ferneres Zusammenarbeiten der Mitglieder der einzelnen Sektionen bis auf weiteres unmöglich machte. Der Ausbruch des Krieges brachte aber nicht nur eine Unterbrechung der gemeinsamen Arbeiten, sondern veranlaßte leider auch Angehörige der Gerbereiwissenschaft und-technik der feindlichen Nationen zu einer aufs schärfste zu verurteilenden Haltung gegenüber den deutschen Fachgenossen. Stets war von deutscher Seite der Anteil, der fremden Nationen an dem Forschungswerk in der Gerbereiwissenschaft zukommt, wohl beachtet und rückhaltslos anerkannt worden. Als im Jahre $1913 \mathrm{H}$. Procter, Professor an der Universität Leeds, wegen vorgerückten-Alters aus' seinem Lehramte ausschied und seine Freunde zur Sammlung von Geldspenden zur Gründung eines internationalen ProcterLaboratoriums aufforderten, das dem englischen Forscher ermöglichen sollte, an seiner bisherigen Wirkungsstätte weiter für die Gerbereiwissenschaft tätig $z u$ sein und das ausländischen Studierenden und wissenschaftlich gebildeten Fachleuten Gelegenheit geben sollte, mit ihm persönlich zusammenzuarbeiten, da wurde in einem von einer Anzahl führender Männer der deutschen Lederindustrie sowie von den Vorständen der deutschen und der österreichischungarischen Sektion des I.V.L. I. C. erlassenen Aufruf der Verdienste Procter's um die Gerbereiwissenschaft mit außerordentlich ehrenden und anerkennenden Worten gedacht und bald auch aus Deutschland eine namhafte Summe als Beitrag für das Procter-Laboratorium nach England überwiesen. Demgegenüber hat Procter sich nach Ausbruch des Krieges nicht enthalten können, ein sehr abfälliges Urteil über die Leistungen der deutschen Gerbereiwissenschaft auszusprechen, das er allerdings später nach einem kräftigen Einspruch der Vorstände der deutschen und österreichisch-ungarischen Sektion des I.V.L.I.C. zurückgenommen hat. Ueberhaupt zeigt sich die auch auf anderen Gebieten 
beobachtete Erscheinung, daß unsere Gegner sich bemühen, die Leistungen der deutschen Chemiker herabzusetzen, indem sie behaupten, daß zumeist Engländer und Franzosen die grundlegenden Arbeiten geschaffen und die Deutschen meist nichts weiter geleistet hätten, als die Forschungsergebnisse der Engländer und Franzosen in die Praxis umzusetzen, auch auf dem Gebiete der Gerbereiwissenschaft und -technik. Ein besonders deutliches Beispiel hierfür zeigt uns J. Paessler ${ }^{1}$, in einem Aufsatze "Zur Geschichte des Ersatzes der Kotbeizen in der Lederindustrie". Er führt darin Stellen aus zwei englischen ${ }^{2}$ ) und einem französischen ${ }^{3}$ ) Fachaufsatz an, in denen ausgesprochen wird, daß die deutschen Chemiker auf dem Gebiete der künstlichen Beizen keine Originalarbeiten geleistet, sondern lediglich die Originalarbeiten fremder Nationen, namentlich der Engländer, ausgenützt hätten. Paessler weist die Unrichtigkeit der französischen und englischen Behauptungen an der Hand der geschichtlichen Entwicklung der künstlichen Beizen nach. $\mathrm{Er}$ führt an, daß wohl einer der ersten, der sich mit dem Ersatz der unsauberen Kotbeizen in der Lederindustrie befaßte, der deutsche Chemiker E. S im on war, der schon 1893 ausgesprochen hat, daß die Wirkung des Hundekotes auf ein Enzym und dessen Stoffwechselprodukte zurückzuführen sei und schon seit 1887 seine auf dieser Anschauung aufgebaute künstliche Beize "Tenuiscum" in den Handel brachte. Dem englischen Chemiker J. T. Wo od kommt dann das Verdienst zu, die Kotbeizen vom bakteriologischen Standpunkt aus eingehend erforscht zu haben. In Deutschland haben Popp und Becker unabhängig von Wood die Kotbeizen in bakteriologischer Hinsicht eingehend erforscht, dann aber mit Wood zusammengearbeitet und als Ergebnis ihrer gemeinsamen Forschungen auf Grund einer Uebereinkunft durch das hygienische Institut von. Popp und Becker die künstliche Beize "Erodin“ in den Handel gebracht, die als wirksamen Bestandteil die im Hundeund Taubenmist befindlichen die Beizwirkung bedingenden Bakterien enthält. Ferner hat $O$. $R \ddot{o} \mathrm{hm}$, und zwar wiederum unabhängig von Wood und von Popp und Becker, ein neues Beizmittel aufgefunden und als Ersatzmittel für Hundekot unter der Bezeichnung "Oropon" in den Handel gebracht, das als wirksamen Bestand-

1) J. P a ess le r, Ledertechn. Rundschau 1916, 65.

2) Leather Trades Review vom 20. Jan. 1915 und Collegium (Londoner Ausgabe) vom 12. Mărz 1915.

3) Halle aux Cuirs vom 3. Jan. 1915. teil das Pankreatin oder Trypsin (Enzym der Bauchspeicheldrüse) enthält. Aus der Geschichte der Kotbeizen ergibt sich daher bei aller Anerkennung der Verdienste Wood's um die Erforschung der Kotbeizen, daß die auf gleichem Gebiet liegenden Forschungen von Popp und $\mathrm{B}$ e cker einerseits und von $\mathrm{Röhm}$ andererseits unabhängig von den Arbeiten Wood's erfolgt sind und daß daher die Versuche, die Verdienste der deutschen Forscher um die Erforschung und Ausgestaltung der künstlichen Beizen zu verkleinern, zurückgewiesen werden müssen.

Es sei in diesem Zusammenhang darauf hingewiesen, daß die für die Lederindustrie so außerordentlich wichtige Erfindung des ersten praktisch brauchbaren durch Synthese gewonnenen künstlichen Gerbstoffes von E. Sti a s ny, einem Oesterreicher, herrührt und von diesem 1913 von England aus zur Zeit seiner Tätigkeit in Leeds der Veröffentlichung übergeben und in Deutschland unter Patentschutz gestellt wurde, während er die Herstellung jenes künstlichen Gerbstoffs der Badischen Anilin- und Sodafabrik in Ludwigshafen am Rhein übertrug, die ein derartiges Erzeugnis unter der Bezeichnung Neradol $D$ in den Handel bringt. Diese Fabrik hat später selbst eine Anzahl Verfahren zur Herstellung und Anwendung neuer synthetischer Gerbstoffe aufgefunden und Patente darauf erhalten. Auch von anderer Seite, z. B. von O. Röh m, wurden neue synthetische Gerbstoffe hergestellt. Derartige künstliche Gerbstoffe, von denen zwei Erzeugnisse der Badischen Anilin- und Sodafabrik, nämlich das schon angeführte Neradol $\mathrm{D}$ und ein später durch Patent geschütztes, unter der Bezeichnung Neradol $\mathrm{N}$ in den Handel kommendes Erzeugnis in der Lederindustrie schon eine ausgedehnte und erfolgreiche praktische Anwendung gefunden haben, sind während des Krieges von erhöhter Bedeutung für unsere Lederindustrie, da sie zur Streckung der während dieser Zeit kaum in zureichender Menge vorhandenen pflanzlichen Gerbstoffe dienen können. Es sind seit Ausbruch des Krieges auch eine Reihe weiterer Neuerungen, Maßnahmen und Vorschläge für die Lederindustrie zu verzeichnen, die der durch den Krieg hervorgerufenen Knappheit namentlich an pflanzlichen Gerbstoffen . abhelfen und die Bestrebungen der Feinde, auch der Lederindustrie Schwierigkeiten $\mathrm{zu}$ machen, zunichte machen sollen. Es handelt sich dabei vor allem um eine bessere Ausnutzung der heimischen Gerberinden Fichtenrinde und Eichenrinde, und anderer bei uns vorhandener gerbstoffhaltiger Pflanzen, sowie der nach deren 
Auslaugung erhaltenen noch gerbstoffhaltigen Rückstände, um einen weitergehenden Ersatz der pflanzlichen Gerbstoffe durch andere in der Lederindustrie (bei der Chromgerbung, Formaldehydgerbung, Chinongerbung usw.) schon angewandte oder neu für die Gerbung vorgeschlagene gerberisch wirksame Stoffe, um den Ersatz tierischer und pflanzlicher Oele und Fette durch andere Stoffe usw.

\section{Arbeiten über die Theorie des Gerbprozesses.}

Es herrscht noch immer ein lebhafter Streit der Meinungen darüber, ob die Gerbung ganz oder in der Hauptsache durch chemische oder durch physikalische Vorgänge bedingt ist. $\mathrm{Zu}$ W. Fahrion, der zwar physikalischen Vorgängen bei der Einleitung der Gerbung eine gewisse Rolle zugesteht, den eigentlichen Gerbvorgang aber als einen chemischen Prozeß erklärt, haben sich in neuerer Zeit weitere Vertreter der chemischen Theorie gesellt, während von den Vertretern der anderen Richtung mehr und mehr kolloidchemische Vorgänge zur Erklärung der Gerbung herangezogen werden.

W. Fahrio n's ${ }^{4}$ ) Gerbetheorie, dessen Veröffentlichung weiter zurückliegt, muß hier mit Bezug auf das Folgende kurz zusammengefaßt angeführt werden. Nach $\mathrm{Fah}$ ri o $\mathrm{n}$ beruht jedë Gerbung auf einer Kondensation zwischen Haut und Gerbstoff. Es ist aber bei dem chemischen Teil der Gerbung einë echte und eine Pseudogetbung zu unterscheiden. Bẹi der echten Gerbung wird zu dem bei der Kondensation abgespaltenen Wasser der Sauerstoff vom Gerbstoff und der Wasserstoff von den basischen Gruppen der Haut geliefert. Bei jeder echten Gerbung findet demnach eine Oxydation der Haut statt. Bei der Pseudogerbung verbindet sich die Haut dagegen nicht unmittelbar mit dem Gerbstoff und bleibt überhaupt völlig unverändert.' Dagegen spaltet der Gerbstoff unter dem katalytischen Einfluf der Hautfasern Wasser ab und geht in ein Anhydroderivat über, das in Wasser schwer löslich oder unlöslich ist und sich daher auf der Haut niederschlägt. Der Unterschied zwischen der echten und der Pseudogerbung besteht demnach darin, daß bei ersterer die Kondensation zwischen Hautfaser und Gerbstoff unmittelbar stattfindet, während bei letzterer der Gerbstoff sich zuerst mit sich selbst und dann mit der Haut kondensiert. Bei der pflanzlichen Gerbung $z$. B. beruht die echte Gerbung

4) Zeitschr. f, angew. Chem. 22, 2083, 2135, 2187, (1909) und Collegium 1910, 16 und Fortsetzungen, sowie S. 249. auf der Wirkung von Chinonen, die durch Hydrolyse in der Gerbstofflösung entstehen, die Pseudogerbung auf der Fällung der Phlobaphene.

Als ein weitererVertreter der chemischen Richtung ist zunächst $O$. S o m m e r h of f zu nennen, der die Gerbung zuerst in der Weise erklärte, daß dabei unter Temperaturerhöhung eine Oxydation des Hautgewebes eintritt, während der Gerbstoff adsorbiert wird und durch seine Gegenwart den Oxydationsprozeß der Haut katalytisch beschleunigt. Später hat jedoch S o $m$ m e r h of f die Annahme einer Oxydation des Hautgewebes fallen lassen und eine "Dehydrationshypothese" über den Gerbvorgang aufgestellt ${ }^{5}$ ). Danach besteht die Gerbung ganz allgemein darin, daß dem Hautalbumin das chemisch gebundene Hydratwasser durch eine durch Gerbstoffe von halboxydiertem Gesamtcharakter bewirkte Wasserabspaltung in rasch aufeinanderfolgendem Oxydations- und Reduktionsproze entzogen wird, wobei als Wasserstoffakzeptor bei der Gerbung mit pflanzlichen Gerbstoffen ein kolloides basisches Phlobaphen, als Sauerstoffakzeptor die komplexen, löslichen, oxydationśfähigen Gerbsäuren dienen. Bei der Chromgerbung stellt das basische kolloide Hydroxyd den Wasserstoffakzeptor, die Gerbsäure, d. h. die Chromischwefelsäure den Sauerstoffakzeptor dar. Für die Sämischgerbung nimmt $S$ om m e rh off an, dah der Wasserstoffakzeptor durch ungesättigte Fettsäureglyzeride, der Sauerstoffakzeptor durch abgespaltene ungesättigte Fettsäuren gebildet wird, die später zu Oxyfettsäuren oxydiert werden. Bei der Chinongerbung wird als Wasserstoffakzeptor das Chinon angenommen, während der Sauerstoffakzeptor sich erst während der Gerbung durch Oxydation des Chinons zu Oxychinon usw. bildet. Bei der Formaldehydgerbung soll der Wasserstoffakzeptor durch Polymerisationsprodukte des Formaldehyds, der Sauerstoffakzeptor durch die im Formaldehyd entweder vorhandene oder während des Gerbvorganges entstehende Ameisensäure gebildet sein.

Auch Powarnin hat verschiedene Gerbtheorien auf chemischer Grundlage entwickelt. Von seiner früheren Anschauung ${ }^{6}$ ), wonach die Gerbung mit pflanzlichen Gerbstoffen, die als phenolhaltige Körper in wässeriger Lösung nach seiner Annahme zwei tautomere Formen, die "Enol"-Form und die "Keto"-Form nebeneinander enthalten, durch die vorhandene und auch während des Gerbprozesses durch Störung des Gleichgewichtes sich stets von neuem

5) O. Som merh of $f$, Collegium 1915, 26.

6) G. Pow a rnin, Collegium 1912, 105. 
bildende "Keto"-Form bedingt sein sollte, ist P ow a r n in zurückgekommen. Er nimmt nunmehr in den pflanzlichen Gerbstoffen ein Karbonyl mit beweglichem Sauerstoffatom, ein „aktives Karbonyl " an ${ }^{7}$ ), das die Gerbstoffe befähigt, bald als Phenol, bald als Peroxyd, Keton, Chinon, Lakton usw. zu reagieren. Das „aktive Karbonyl " soll ferner die Ursache von Kondensationserscheinungen, z.B.der Phlobaphenbildung, sein und auch die gerbende Wirkung bedingen, indem es unter Abspaltung von Sauerstoff in Form von Wasser mit den verschiedenen Amingruppen der Haut reagiert.

Vom Standpunkt seiner neueren Theorie wendet sich Powarnin gegen die Anschauung Fahrion's, daß sich beim Stehenlassen von Lösungen pflanzlicher Gerbstoffe unter dem Einflusse des Wassers Peroxyde bilden und diese, deren Bildung beim Gerben infolge der schwach alkalischen Reaktion der Haut noch begünstigt wird, die Ursache der gerberischen Wirksamkeit seien, indem die stickstoffhaltigen Gruppen der Haut mit dem aktiven Sauerstoff der Chinone und Peroxyde in Reaktion treten. Powarnin weist hierzu darauf hin, daß Gerbstoffe wohl in alkalischer Lösung, dagegen in neutraler Lösung nur in geringem Maße und in saurer Lösung überhaupt keine Chinone bilden. Die alkalische Reaktion der Haut kommt bei der Chinongerbung nicht in Betracht, da sich die Gerbung auch in saurer Lösung vollzieht, wenn die "Alkalität" der Haut durch Bildung von "Azidoglutin" aufgehoben ist. Wenn das Wasser bei der Gerbung eine Rolle spielt, so sollte eine Gerbung in al koholis cher Lösung ausgeschlossen sein, während sowohl von ihm als auch von $\mathrm{F}$ a h r i o n selbst ausgeführte Versuche zeigten, daß Gerbstoff auch in alkoholischer Lösung von Hautpulver adsorbiert wird. Gegen die Annahme der Wirksamkeit von Peroxyden bei der Gerbung spricht auch die praktische Erfahrung, daß gerade Umstände, die die Bildung von Peroxyden begünstigen, z. B. Liegenlassen an der Luft und dem Licht, eine Verschlechterung des Leders bewirken können. Powa rn in schließt daraus, daß Peroxyde, wenn sie überhaupt gebildet werden, nicht die Ursache der Gerbung sein können.

$\mathrm{Zu}$ diesen Ausführungen Powarnin's bemerkt $\mathrm{Fahrion}$ in einer Erwiderung ${ }^{8}$ ), daß er den Einwand Powarnins', daß die Peroxyde sich in Lösungen pflanzlicher Gerbstoffe nur in geringer Menge bilden, nicht als

7) G. Powarn in, Collegium 1914, 633.

8) W. Fahrion, Collegium 1914, 707. stichhaltig anerkennen könne, da geringe Mengen sich summieren können, wenn sie im Entstehungszustande mit der Haut in Reaktion treten. Gegenüber dem Hinweis, daß in saurer Lösung keine Peroxyde gebildet werden, sei zu bemerken, daß die vorwiegend alkalische Haut eine be trächtliche Menge Säure durch Neutralisation unschädlich machen könne. Dagegen sei der Einwand, dab die Gerbung mit pflanzlichen Gerbstoffen auch in alkoholischer Lösung möglich sei, nicht ohne weiteres abzuweisen. Fahrion gibt mit Bezug hierauf $z \mathfrak{u}$, daß das von ihm beigebrachte Untersuchungsmaterial auch in anderer Weise erklärt werden könne, derart, daß nur der auf die Kondensationsbildung sich beziehende Teil seiner Theorie Geltung behalten würde.

Bei einer Besprechung der Theorie Sommerhoff's sagt Fahrion, dab diese zur Erklärung der verschiedenen Gerbarten, namentlich aber der Sämischgerbung ungeeignet sei. Bei der Sämischgerbung üben nicht Fettsäureglyzeride, sondern die ungesättigten, freien Fettsäuren die gerbende Wirkung aus, während das Glyzerin der Fettsäureglyzeride keine Rolle spielt. Die ungesättigten, freien Fettsäuren werden außerdem lediglich oxydiert, nicht auch, wie es die Theorie Sommerhoff's erfordert, teilweise reduziert, da die Menge der im Tran enthaltenen ungesättigten Fettsäuren sich bei der Sämischgerbung nicht vermehrt.

Eine Gerbetheorie, die völlig auf kolloidchemischer Grundlage beruht, hat W. Mölle r ${ }^{9}$ ) entwickelt. Er erklärt die Eigenschaften der Gerbstofflösungen und die Gerbvorgänge aus Peptisationserscheinungen. Er führt aus, daß wahre Lösungen niemals gerbende Eigenschaften zeigen können, sondern nur peptisierte Sole und betrachtet als Ursache der Peptisationserscheinungen bei den Gerbstofflösungen die Bildung komplexer Verbindungen zwischen dem Peptisator und der peptisierten Substanz, wobei er annimmt, daß der Peptisator zur peptisierten Substanz in einem nahen Verwandtschaftsverhältnis stehen muß. Bei den pflanzlichen Gerbstoffen ist die peptisierte Substanz bei den Pyrogallolgerbstoffen die Ellagsäure, bei den Protokatechingerbstoffen ein Phlobaphen, während der Peptisator bei beiden Gerbstoffarten der gleiche ist und zwar das Tannin. Der Unterschied, zwischen den Pyrogallol- und Protokatechingerbstoffen besteht darin, dab die ersteren im Verhältnis mehr Tannin als Ellagsäure, also einen gewissen

9) W. Möller, Collegium 1915, 49, 193, 225 u. 253. 
Ueberschuß des Peptisationsmittels, die letzteren überwiegend Phlobaphene und daneben nur geringe Mengen von Tannin enthalten und durch letzteres nur teilweise peptisiert sind. Bei den zur Mineralgerbung verwendeten Lösungen entsteht der Peptisationszustand dadurch, daß den stark dissoziierten Lösungen bei Berührung mit der Haut ein Teil des Säureanteils durch Adsorption entzogen wird und daß das in Gelform übergehende Metallhydroxyd im Entstehungszustand durch das gleichzeitig gebildete saure Salz desselben Metalls peptisiert und unter Bildung einer homogenen Lösung in ein Sol übergeführt wird. Die wässerige Formaldehydlösung für die Formaldehydgerbung ist ein Sol, in welchem die in Formaldehydlösungen stets enthaltenen Polymerisationsprodukte des Formaldehyds durch das einfache Formaldehyd peptisiert sind. Die bei der Chinongerbung verwendeten Lösungen stellen Sole dar, bei denen das Chinon durch das daneben stets vorhandene durch Reduktion gebildete Hydrochinon peptisiert ist. Wie die Gerbstofflösung, so wird auch die Haut erst zur Gerbung befähigt, wenn sie sich im Zustande der Peptisation befindet. Die Ueberführung in diesen Zustand wird durch die der Gerbung vorhergehenden vorbereitenden Arbeiten bezweckt und erreicht, indem dabei die gegen Peptisatoren weniger widerstandsfähige Zwischenzellsubstanz völlig peptisiert wird, während bei sachgemäßer Arbeit die widerstandsfähigeren Hautfibrillen nur in ihren äußeren Schichten in den Zustand der Peptisation versetzt werden. Der Gerbprozeß besteht in der Aufhebung des Solzustandes der peptisierten Lösung durch die Hautsubstanz. und in einer Trennung des Peptisators von der peptisierten Substanz auf dem Wege der Osmose. Hierbei wirkt die Substanz der Hautfasern als Dialysator und deren Oberfläche als halbdurchlässige Membran. Die Hautsubstanz entzieht dem Sol den Peptisator, der in die Haut diffundiert und mit dieser eine feste Lösung bildet, während das Gel sich ausscheidet und die Hautfasern in Form von Mikrokristallen umlagert. Die Eigenschaften des Leders sind in erster Linie von der Menge der abgelagerten peptisierten Substanz abhängig.

W.Fahrion ${ }^{10}$ ) bezeichnet die Anschauungen Möller's über das Wesen der Gerbung als eine Rückkehr zur K n a p p'schen Umkleidungstheorie. Er weist darauf hin, daß die Interzellularsubstanz bei der Gerbung keine wesentliche Rolle spielen könne, da sie nur in geringer Menge vorhanden sei und nach B. Lier aus

10) W. Fahrion, Collegium 1915, 332. mukoiden Stoffen besteht, demnach bei den Vorarbeiten des Aescherns usw. sicher völlig beseitigt wird. Er bestreitet die Behauptung Mölle r's bei der Erklärung der Formaldehydgerbung, daß man mit reinem Formaldehyd überhaupt nicht gerben könne, sondern nur mit Formaldehydlösung, die als peptisierte Substanz Paraldehyd enthält. Die ungenügende Qualität des mit Formaldehyd gegerbten Leders ist dadurch bedingt, daß der Paraldehyd die Phlobaphene als Aufpolsterungsmittel nicht ersetzen kann. Stias ny habe die Formaldehydgerbung mit seiner physikalischen Gerbetheorie auch nicht erklären können und habe auch dem Paraldehyd keinerlei Bedeutung beigemessen. Gegenüber der Aninahme Mölle r's, daß das unter der Einwirkung des Wassers aus dem Chinon entstehende Hydrochinon als Peptisator und das Chinon als peptisiertes Gel, wirke, weist $\mathrm{Fah} r$ i on auf seine Untersuchungsergebnisse hin, wonach eine alkoholische Chinonlösung auch im Dunkeln gerbt, wobei neben hochmolekularen Derivaten allerdings auch Hydrochinon entsteht, aber nur sekundär und infolge Einwirkung der Haut auf das Chinon. Es sei jedoch durch nichts gerechtfertigt, diese Vorgänge als Peptisation zu bezeichnen. Fah rion vermißt ferner bei den Ausführungen Möller's Angaben darüber, welches bei der Chromgerbung nach dem Zweibadverfahren der Peptisator sei. Er weist darauf hin, daß die Theorie Möller's keine rein physikalische sei und der chemischen Theorie durchaus nicht fern stehe, da er die Peptisation mit P. P. von Weimarn auf eine Komplexbildung nach Art des Chinhydrons zurückführe, dessen Bildung aber auch auf chemischer Affinität beruhe. Wenn man mit Mölle $r$ annähme, daß derartige Komplexverbindungen sich zwischen Tannin und Phlobaphenen bilden, so sei es auch nicht ausgeschlossen, daß auch die Haut mit den Phlobaphenen ähnliche Komplexverbindungen eingeht, denn die Forderung Möller's, daß der Peptisator zu der peptisierten Substanz in einem nahen Verwandtschaftsverhältnis stehen müsse, sei eine rein willkürliche Annahme. W. Mölle $\mathrm{r}^{11}$ ) erwidert Fahrion, daß die Interzellularsubstanz bei den vorbereitenden Arbeiten wohl peptisiert wird, aber, da sie die Hautfibrillen umlagert, nicht völlig entfernt werden kann, ohne letztere selbst anzugreifen, was bei sachgemäßer Behandlung der Haut vermieden wird. Zur Formaldehydgerbung bemerkt $M$ öll e r, daß Formaldehydlösungen stets wesentliche Mengen von Paraldehyd enthàlten

\footnotetext{
ii) Collegium 1915, 356 .
} 
und daß er an seiner Auffassung festhalten müsse, daß die Formaldehydlösung des Handels bereits eine peptisierte Lösung von Kondensationsprodukten des Formaldehyds darstellt. Außerdem sei $z u$ beachten, daß die bei der Vorbereitung der Haut gebrauchten Stoffe, wie Säuren und Alkalien bei Berührung mit der Formaldehydlösung leichtKondensationsprodukte des Formaldehyds erzeugen können. Die Tatsache, daß Paraldehyd die Phlobaphene nicht $z u$ ersetzen vermag, erklärt $M$ öll e r einerseits mit dem geringen Molekulargewicht des Formaldehyds und seiner Polymerisationsprodukte, andererseits damit, daß nach seiner Theorie die Menge der ausgeschiedenen peptisierten Substanz auch von dem Lösungsvermögen des Peptisators in der Haut abhängt und um so geringer ist, je weniger von dem Peptisator in die Haut diffundiert. Man erhält daher eine wesentlich bessere Wirkung bei der Formaldehydgérbung, wenn das Aufpolsterungsvermögen des Paraldehyds in der Formaldehydlösung wie z. B. bei der Weins chenk'schen FormaldehydNaphtholgerbung durch andere peptisierte Substanzen aufgebessert wird. Auch bei der Chinongerbung hält Möller seine Anschauungen aufrecht, daß die Bildung der Kondensationsprodukte aus dem Chinon als eine Peptisation zu bezeichnen sei und daß dabei eine komplexe Verbindung zwischen peptisierter Substanz und Peptisator eintritt. Zur Erklärung der Zweibadchromgerbung bemerkt $M o ̈ 11 \mathrm{er}$, daß das bei der Reduktion im Zweibad entstehende Chromoxyd zusammen mit der durch Oxydation der zunächst gebildeten schwefligen Säure entstehenden Schwefelsäure Chromsulfat bildet. Der dann folgende Vorgang der Peptisation ist der gleiche wie bei der Gerbung mit Alaun und anderen Mineralsalzen. Von dem Einbadverfahren unterscheidet sich das $Z$ weibadverfahren nur dadurch, daß bei letzterem die peptisierte Lösung von Chromoxyd unmittelbar auf der Faser erzeugt wird, während bei dem Einbadverfahren mit der fertig gebildeten peptisierten Lösung gearbeitet wird.

\section{Arbeiten über die Rohhaut, deren Konservierung und Desinfizierung.}

Gewisse unter bestimmten Bedingungen eintretende Veränderungen der Rohhaut, die meist mit Färbungen verbunden sind, faßt man unter der Bezeichnung "Salzflecken" zusammen. Die Salzfleckenfrage ist nicht nur in gerberischer sondern auch in volkswirtschaftlicher Beziehung von Bedeutung, da die Rohhäute durch Salzflecken in ihrem Werte meist wesentlich vermindert, vielfach für eine gerberische Verwertung unbrauchbar werden. G. $\mathrm{Abt}^{12}$ ) hatte früher gefunden, daß das Auftreten gewisser Salzflecken durch die Gegenwart von Kalziumsulfat (Gips) bedingt ist, das sich vielfach als Verunreinigung im Häutesalz vorfindet. Das bei der durch Bakterien bewirkten Zersetzung der Haut sich bildende Ammoniumphosphat setzt sich mit dem Kalziumsulfat zu Kalziumphosphat um, das dann aus der Haut ausschlägt. Durch die Gegenwart von Spuren von Eisen wird das Auftreten der Salzflecken begünstigt. Auch eine Mitwirkung von Bakterien hält Abt für wahrscheinlich. Später ${ }^{13}$ ) gelang es $\mathrm{Abt}$, auf peptonisierter Gelatine eine Bakterienart zu isolieren, deren braun gefärbte Kolonien sich bei Zusatz von Kalziumsulfat und noch mehr von Spuren von Eisensalz dunkler färben. Weitere Versuche ergaben, daß die färbende Substanz sich auf Kosten der Hautlymphe und des Protoplasmas der Epidermis bildet und sich auf dem Niederschlag von Kalziumphosphat und etwa vorhandenen Spuren von Eisensalz absetzt. Der Farbstoff zeigt nach seinem Verhalten Aehnlichkeit mit den Melaninen. A b t hält es nicht für ausgeschlossen, daf auch andere zur gleichen Art gehörige Bakterien ähnliche Parbstoffe zu bilden vermögen. W. Eitn e ${ }^{14}$ ) hat die Versuche von $\mathrm{A} b$ t unter Verwendung von Kalziumsulfat enthaltendemAbraumsalz wiederholt,konnte jedoch die von $\mathrm{Abt}$ angegebenen Erscheinungen nicht beobachten. In dem zweiten Bericht ${ }^{15}$ ), der vom Internationalen Verein derLederindustrieChemiker eingesetzten Kommission zum Studium der Frage betreffend Konservierung und Desinfektion von Häuten und Fellen hält jedoch $A$ b t auf Grund weiterer Versuche seine Meinung, dab die Bildung der von ihm untersuchten Salzflecken bei Mitwirkung von Bakterien an die Gegenwart von Kalziumsulfat gebunden sei, aufrecht. Andererseits wiederholt $\mathrm{H}$. B e cker, Frankfurt am Main, an gleicher Stelle seine frühere Feststellung ${ }^{16}$ ) auf Grund eingehender bakteriologischer Forschungen, daB als eigentliche Ursache der von ihm untersuchten farbigen Flecken die Tätigkeit bestimmter von ihm isolierter Bakterien anzusehen sei, wenn, auch im Wirkungsbereich der letzteren Stoffe nachgewiesen werden können, die durch Verbindung

\footnotetext{
12) G. Abt, Collegium 1912, 388 .

13) G. A bt, Collegium 1913, 204.

14) W. Eitner, Der Gerber 1913, 114.

15) Collegium 1914, 267.

16) Collegium 1912, 408.
} 
der Abbauprodukte des Hauteiweißes mit Verunreinigungen des Kochsalzes entstanden sind. J. H. Yo ku m ${ }^{17}$ ) hat bei Hautflecken von rostbrauner oder brauner Farbe festgestellt, daß diese stets Eisen enthalten und führt die Entstehung jener Flecken darauf zurück, daß das bei Zersetzung des in der Haut enthaltenen bez. dieser als Verunreinigung anháftenden Blutes durch Umwandlung des Hämoglobins in Hematin freiwerdende Eisen in der Haut fixiert wird. Kohnstein ${ }^{18}$ ) weist auf die nach dem Tode der Tiere infolge von Zersetzung der Haut und dadurch hervorgerufener Veränderung der Pigmente vielfach auftretenden blauschwarzen Färbungen hin. Da es ihm gelang, ganz ähnliche blauschwarze Färbungen durch Salzen der Häute mit Blut enthaltendern Kochsalz künstlich zu erzeugen, so nimmt er an, daß das Blut bez. dessen Zersetzungsprodukte bei der Entstehung solcher Färbungen eine Rolle spielen. Daß das Auftreten von Salzflecken auch von der Art des Vergällungsmittels des zur Konservierung verwendeten Salzes abhängt, wurde schon früher von verschiedenen Seiten nachgewiesen. So fand $\mathrm{Pa}$ essler ${ }^{19}$ ), daß bei Verwendung von Soda als Vergällungsmittel weniger Salzflecken auftreten als bei Petroleum. Gänzlich verfehlt ist aber der Vorschlag von C. Schiffk or $n^{20}$ ), dem Häutesalz zur Vergällung 5 Proz. gebrauchte alte Aescherbrühe zuzusetzen, da dabei auch die in dieser in beträchtlichem Maße enthaltenen Bakterien und Zersetzungsprodukte in das Häutesalz und damit auf die Haut gebracht werden und daher beim Salzen mit einem in dieser Weise vergällten Salz dessen konservierende Wirksamkeit herabgesetzt und die Entstehung von Salzflecken mehr befördert als verhütet werden würde. Nach L. Lloyd ${ }^{21}$ ) ist die Bildung gewisser Flecken auf der Haut auf die verflüssigende Wirkung aeorober Bakterien zurückzuführen und wird durch die Gegenwart von Blut begünstigt. $\mathrm{Er}$ empfiehlt zur Verhinderung der Zersetzung der Haut irgendwelche antiseptisch wirkende organische Verbindungen, $z$. B. organische Arsenverbindungen zu verwenden.

C. R o $\mathrm{m}$ a $\mathrm{n}$ a und G. B a I d r a c co ${ }^{22}$ ) schlieBen aus ihren Versuchen, daß die Salzflecken in der Hauptsache durch in den Häuten befind-

17) J. H. Yokum, Journ. Amer. Leather Chemists Association 1913, 22.

18) B. Kohnstein, Collegium 1913, 587.

19) J. P a essler, Ledertechn. Rundschau 1912, 140.

${ }^{20}$ C. Schiffkorn, Der Gerber 1915, 279.

21) L. L lo yd, Collegium 1913, 193.

22) Collegium 1914, 517. liche Verunreinigungen bedingt sind und sich durch gutes Waschen vor dem Salzen vermeiden lassen. Durch einen Zusatz von Natriumfluorid oder auch von Borax wird die konservierende Wirkung des Häutesalzes wesentlich erhöht.

Von großer Bedeutung ist in wirtschaftlicher wie in gesundheitlicher:Beziehung die Desinfizierung der Häute, die Zerstörung etwa darin vorhandener gesundheitschädlicher Keime, vor allem der Milzbrandkeime.

Von den zahlreichen zur Desinfektion der Rohhäute vorgeschlagenen Verfahren haben zwei aus neuerer Zeit eine besondere Beachtung gefunden: Das Verfahren von SeymourJones ${ }^{23}$ ), wobei die Häute mit einer 0,02 Proz. Sublimat und 1 Proz. Ameisensäure enthaltenden Flüssigkeit behandelt werden und das Verfahren von $\mathrm{Schattenfroh-Kohnstein}{ }^{24}$ ), das als Desinfektionsmittel eine 2 Proz. Salz und 10 Proz. Kochsalz enthaltende „Pickel"- Flüssigkeit benutzt. Die Wirksamkeit der beiden Verfahren ist von verschiedenen Seiten nachgeprüft worden. Schnürer-Sevčik ${ }^{25}$ ) fanden, daß die von Seymour-Jones angegebene Stärke der Sublimatflüssigkeit nicht gentigt, sondern daß zur sicheren Zerstörung der Milzbrandkeime eine 0,2 Proz. Sublimat und 1 Proz. Ameisensäure enthaltende Flüssigkeit angewendet werden muf. Nach W. Tille y ${ }^{26}$ ) wird aber schon bei einer Erhöhung des Sublimatgehaltes auf 0,04 Proz. eine genügende Wirksamkeit erzielt, falls die Haut binnen ein bis zwei Wochen nach der Desinfizierung nicht mit Stoffen in Berührung kommt, die, wie z. B. das beim Weichen und Aeschern zum Teil mit verwendete Schwefelnatrium, die Wirkung des Sublimates aufheben. Auch G. $\mathrm{Abt}^{27}$ ) fand, daß die Wirksamkeit des Verfahrens von S e y m o u r-J o n e s bei späterer Behandlung der Häute mit Schwefelnatrium herabgemindert wird, dagegen nicht bei Haarlockerung unter Verwendung, von Kalk oder Schwefelarsen. Dagegen haben H. Reichel und V. Gegenbau er ${ }^{28}$ ) mit dem Verfahren von Seymour-J on es überhaupt keine guten Ergebnisse erhalten. Durch das Verfahren von Schattenfroh-Kohnstein können nach

${ }^{23}$ ) Seymour-Jones, The Leather Trades Review vom 4. Jan. 1911 und Referat im Collegium 1911, 106. a4) Schattenfroh-Kohnstein, Collegium 1911, 248 und 304.

$\left.{ }^{25}\right)$ Sch nürer-Sevěi k, Häute und Leder 1913, 18.

26) W.Tilley, Journ. of The American Leather Chemists Association 1916, 131.

27) G. A bt, Collegium 1914, 277.

$\left.{ }^{28}\right) \mathrm{H}$. Reichel und V. Gegenbauer, Collegium 1913,435 
Sch n ür e r - S e v č i k ${ }^{29}$ ) bei dicken Rindshäuten Milzbrandkeime selbst nach 72 stündiger Einwirkungsdauer des Salzsäure-Kochsalzgemisches nicht sicher abgetötet werden, während die Desinfektion leichterer Felle geringere Schwierigkeiten bietet. G. $\mathrm{Abt}^{30}$ ) fand das Verfahren von Schattenfroh-Kohnstein an sich genügend wirksam, weist aber gleichzeitig darauf hin, daß dieses Verfahren nur bei Häuten anwendbar ist, die gepickelt werden dürfen, also bei solchen, die zu weichen Ledersorten verarbeitet werden. Mit diesem Verfahren haben auch Reichel und Gegenbauer ${ }^{31}$ ) gute Ergebnisse erzielt. $\mathrm{Abt}^{32}$ ) fand, dab auch Chlor die Milzbrandkeime abtötet; doch ergeben sich bei Anwendung dieses Desinfektionsmittels praktische Schwierigkeiten, da die Hypochlorite bez. der Chlorkalk einen sehr schwankenden Gehalt an wirksamem Chlor besitzen und letzterer vorher in jedem Fall bestimmt werden müßte. Nach Tilley ${ }^{33}$ ) werden Milzbrandkeime, auch die Sporen, durch eine 2,5 prozentige Formaldehydlösung getötet, dabei aber gleichzeitig die Häute in einer für die Gerbung ungünstigen Weise verändert, während sich eine fünfprozentige Phenollösung gegen Milzbrandsporen als völlig unwirksam erweist. C. S chiffkorn ${ }^{34}$ ) empfiehlt die Häute zur Desinfektion mit einer drei- bis vierprozentigen Lösung von Rohkresol zu bespritzen.

Bei gewissen trocknen Häuten, die aus Ostasien stammen, findet sich auf der'Fleischseite ein erdiger Belag, der zur Konservierung dienen soll, vielfach aber auch zur Erhöhung des Gewichtes und zur Verdeckung von Hautfehlern benutzt wird. Kohnstein ${ }^{35}$ ) erhielt für den Belag derartiger trockner Häute, abgesehen von den orga nischen Stoffen, folgende Untersuchungsergebnisse: Bei ostindischen Kipsen 11,8 Proz. Feuchtigkeit, 36,1 Proz. Mineralstoffe, die in der Hauptsache aus Kalkverbindungen, Magnesiumsulfat (14,8 Proz.) und Alkaliverbindungen bestehen, bei trocknen ostindischen Ziegenfellen 8,7 Proz. Feuchtigkeit, 31,7 Proz. Mineralstoffe, 1,3 Proz. Kaliumsulfat, 12,9 Proz. Magnesiumsulfat und 32,2 Proz. Natriumsulfat $\left(\mathrm{Na}_{2} \mathrm{So}_{4}+\right.$ $10 \mathrm{H}_{2} \mathrm{O}$ ). C. $\mathrm{Lamb}^{36}$ ) fand für den Belag bei

$\left.{ }^{29}\right)$ Schnürer-Sevъrik, a. a. O.

so) G. Abt, a. a. O.

31) H. Reichel und V. Gegenbauer, a. a. O.

32) G. A bt, a. a. O.

33) W. Tilley, a. a. O.

34) G. Schiffkorn, a. a. O.

${ }^{35}$ ) B. Kohnstein, Allgemeine Gerberzeitung $1913,27$.

${ }^{36}$ ) C. La m b, Collegium 1914, 357. ostindischen Ziegenfellen folgende Zusammensetzung: 8,1 Proz. Kieselsäure und Sand, in der Hauptsache Natriumsulfat und geringe Mengen der Sulfate und Chloride von Kalium, Eisen, Kalk und Magnesia, sowie Spuren von Phosphorsäure und Kohlensäure. J. L a e m m l e ${ }^{37}$ ) hat festgestellt, daß die amerikanischen Häutehändler zur Beschwerung ihrer Häute vielfach schwefelsaure Tonerde verwenden.

\section{Arbeiten über das Weichen, Aeschern und Schwitzen.}

Zum Weichen trockner Häute wird neuerdings ein Zusatz von Borax zum Weichwasser besonders empfohlen ${ }^{38}$ ), zumal dieser auch gleichzeitig fäulnishemmend wirkt, und wegen seiner schwach alkalischen Reaktion die Häute unmittelbar aus der Weiche in den Aescher gebracht werden können. Von anderer Seite wird die Ameisensäure als ein gutes Weichmittel angegeben ${ }^{39}$ ).

Die Wirkungsweise des Schwitzens und Aescherns behandelt W. Eitne ${ }^{40}$ ). Diese ist bei beiden Verfahren insofern gleich, als dabei die Schleimschicht zerstört und dadurch die Haarlockerung erzielt wird, andrerseits dadurch verschieden, daß beim Schwitzen eine Zerlegung der Bestandteile der Schleimschicht in einfachere Verbindungen und gleichzeitig eine Verflüssigung stattfindet, während beim Aeschern die Stoffe der Schleimschicht unmittelbar aufgelöst werden und sich mit den alkalischen Stoffen verbinden. Der Kalkäscher löst aber nicht nur die Schleimschicht auf, sondern bewirkt auch gleichzeitig durch Erhöhung der Wasseraufnahmefähigkeit der Haut deren Schwellung. Im alten Kalkäscher tritt zur Wirkung des Kalkes noch diejenige von Bakterien. Im Gegensatz zu Eitner, der die haarlockernde Wirkung des Kalkäschers in der Hauptsache der lösenden Wirkung des Kalkes zuschreibt, kommt $G$. B e n ne ${ }^{41}$ ) auf Grund von Versuchen zu dem SchluB, daß die haarlockernde Wirkung des Kalkes bei den gewöhnlichen Aescherverfahren außerordentlich gering ist, daß die Haarlockerung vielmehr fast ganz durch die chemische Wirkung von Enzymen unter Mitwirkung von Bakterien zustande kommt, wobei die Enzyme die weicheren Keratine und

$\left.{ }^{37}\right)$ J. L a e m m le, Journ. of The American Leather Chemists Association 1915, 351.

$\left.{ }^{38}\right)$ Ledertechn. Rundschau 1914, 292.

${ }^{39}$ ) Le Cuir 1914, 446 u. Referat Collegium 1915, 343.

$\left.{ }^{40}\right)$ W. Eitner, Der Gerber, 225.

$\left.{ }^{41}\right)$ G. Bennet, Journ. of The American Leather Chemists Association 1915, 569. 
die Haarwurzeln lösen. Der Kalk trägt im wesentlichen nur dadurch zur Haarlockerung bei, daß er erweichend wirkt und dadurch die Einwirkung der Enzyme erleichtert.

Mit Bezug auf die praktisch anzuwendende Aescherdauer herrscht allgemein die Ansicht, daß die für Oberleder bestimmten Häute länger geäschert werden sollen als diejenigen für Sohlleder. W. James und R. Parker ${ }^{42}$ ) kamen jedoch bei Versuchen zu Ergebnissen, nach denen diese Anschauung nicht unbedingte Gültigkeit besitzt. Sie fanden, daß einerseits Häute für Sohlleder bei einer gegenüber der sonst üblichen wesentlich verlängerten Aescherdauer (14-18 Tage) ein gutes Rendement ergaben und daß andrerseits auch bei der sonst bei Sohlleder üblichen kurzen Aescherdauer gutes Oberleder erzielt werden kann.

Vielfach herrscht die Anschauung, daB die Menge des überschüssigen Kalkes im Aescher auf das Aescherergebnis ohne Einfluß ist. A. Rog e rs ${ }^{43}$ ) führte eine Anzahl von Versuchen auf praktischer Grundlage durch, um bei Gegenwart verschiedener Mengen Kalk im Aescher die Wirkung des Kalkes auf die Schwellung und dessen Aufnahme durch die Haut festzustellen. Es wurde gefunden, daß eine größere Menge Kalk eine größere Gewichtszunahme der Rohhäute und auch eine erhöhte Kalkaufnahme der Haut zur Folge hat. Weiter ergab sich, daß der Verlust an Hautsubstanz bei Verwendung eines frischen Aeschers gröber ist, als bei einem schon gebrauchten und „zugebesserten "Aescher. Von der Gewichtszunahme der Häute kommen 80 Proz. auf den ersten Tag, während die Aufnahme des Kalkes in der Hauptsache während der ersten zwei Tage erfolgt. Um die geringere hautlösende Wirkung des alten Aeschers gegenüber dem frischen Aescher zu erklären; nimmt Rogers an, daß die im alten Aescher enthaltene Aescherflüssigkeit wie ein Schutzkolloid wirkt.

T. Wood ${ }^{44}$ ) stellt auf Grund seines früher mit J. Law erstatteten Berichtes über die "Aescherkontrolle bei Oberleder" ${ }^{45}$ ) fest, daß der Stickstoffgehalt mit dem Alter des Aeschers zunimmt; doch wird am Anfange des Aescherns die größte Menge an Hautsubstanz aufgelöst. Die Höchstgrenze, bis zu der der Gehalt des

${ }^{42}$ ) W. James u. R. Parker, Collegium (Londoner Ausgabe) 1915, 136.

4) A. Rogers, Journ. of The American Leather Chemists Association 1914, 42.

44) T. Wood, Collegium 1914, 308.

${ }^{45}$ ) Collegium 1912, 121 .
Aeschers an Stickstoff gehen kann, bevor die Aescherbrühe verworfen werden muß, ist schwierig zu bestimmen, da hierbei eine ganze Anzahl vori Faktoren in Betracht kommt, namentlich die Temperatur, der Grad der Zersetzung der vorhandenen Proteide und die Zahl der anwesenden proteolytischen Bakterien; doch nimmt Wood an, daf im allgemeinen ein Gehalt der Aeșcherbrühe von bis $\mathrm{zu} 0,2 \mathrm{~g}$ Stickstoff im Liter nicht schädlich wirkt.

R. B locke ${ }^{46}$ ) gibt eine Kurve an, die den während des Aescherns eintretenden Verlust an Hautsubstanz einer Partie Häute und eine andere, die den Gehalt an Hautsubstanz im Aescher während dessen Verwendungszeit zeigt. Aus der ersteren ergibt sich, daß bei zehntägigem Aeschern 1 Proz. der Hautsubstanz gelöst wird, aus der letzteren, daß ein Aescher, in dem unausgesetzt Häute geäschert werden, nach 10 Tagen $3 \mathrm{~g}$ Hautsubstanz im Liter enthält.

Ein neues, durch D. R. P. Nr. 268873 geschütztes Verfahren zum Enthaaren von Häuten und Fellen hat $\mathrm{O}$. $\mathrm{Röh}{ }^{47}$ ) aufgefunden. Es gründet sich auf dessen Beobachtung, daß die Häute sich enthaaren und vom Schmutz befreien lassen, wenn man sie mit einer zweckmäßig alkalisch gemachten Lösung von Tryptase, am besten Pankreastryptase, behandelt. Ein derartiges Verfahren könnte eine bedeutende Vereinfachung der zur Ueberführung der Haut in Blöße nötigen Arbeiten herbeiführen, da dabei die Behandlung der Haut mit Kalk oder Sulfiden sowie die zur Entfernung dieser Stoffe nötigen Arbeiten in Fortfall kommen würden.

$\mathrm{R}$ ö $\mathrm{h} \mathrm{m}$ hat die bei seinem Aescherverfahren wirksamen Enzyme „Arazym" und das Verfahren selbst „Ara"-Aescher genannt. Nach seinen Angaben hat sich das Verfahren bereits gut in die Lederindustrie eingeführt. In Amerika hat A. Roger s ${ }^{48}$ ) mit dem Arazym günstige Ergebnisse erhalten. Da er jedoch den Erfinder des Aescherverfahrens nicht nennt, so nimmt Röhm nochmals Gelegenheit, ausdrücklich auf seine Urheberschaft der Erfindung hinzuweisen ${ }^{49}$ ).

Mit Bezug auf die Bestimmung von Alkalisulfiden in Aescherbrühen hatten Blockey und Meh $\mathrm{d}^{50}$ ) vorgeschlagen die dafür von Procter verwendete ammoniakalische Zinkvitriollösung durch eine Chlorammonium enthaltende Zink-

46) R. B lockey, Collegium 1913, 629.

47) O. Röh m, Patentschrift s. Collegium 1914, 86.

$\left.{ }^{45}\right)$ A. Ro gers, nach Leather Trades Review 1915, 281 und Referat Collegium 1916, 122.

49) Collegium 1916, 151.

${ }^{50}$ J. R. B lockey und V. Mehd, Coll. 1912, 300. 
vitriollösung zu ersetzen, da andernfalls durch Mitfällung von Zinkhydroxyd Fehler entstehen. D. Mc Candlish und A. Wils on ${ }^{51}$ ) haben beide Lösungen zur Untersuchung von Flüssigkeiten, die Sulfide und Sulfide neben Kalk enthalten, benutzt und dabei gefunden, daß weder die eine noch die andere Lösung unter allen Umständen genaue Ergebnisse liefert. Sie konnten auch mit der später von Blockey und $M$ ehd angegebenen Titrationsflüssigkeit, die neben Zinkvitriol und Chlorammonium noch Ammoniak enthält, keine befriedigenden Ergebnisse erhalten. Zur Bestimmung des nutzbaren Kalkes in Aescherbrühen, die neben Kalk auch Schwefelnatrium enthalten, verfährt J. Helfrich ${ }^{22}$ ) in folgender Weise: Ein bestimmtes Volum der filtrierten und dann verdünnten Aescherbrühe wird mit $\frac{n}{10} \mathrm{H}_{2} \mathrm{SO}_{4}$ zunächst mit Phenolphthalein bis zum Farbenumschlag, dann mit Methylorange zu Ende titriert. DerUnterschied zwischen den beiden Titrationsergebnissen in $\mathrm{ccm}$ entspricht der Hälfte des vorhandenen Schwefelnatriums. Zieht man von dem bei Phenolphthalein erhaltenen Titrationsergebnis eine dem Unterschied zwischen den mit den beiden Indikatoren erhaltenen Ergebnissen entsprechende Anzahl ccm ab, so wird auch das in der Lösung befindliche Aetznatron berücksichtigt. Die noch verbleibende Alkalinität in $\mathrm{ccm}$ entspricht dem nutzbaren Kalk. $1 \mathrm{ccm} \frac{\mathrm{n}}{10} \mathrm{H}_{2} \mathrm{SO}_{4}=0,0028 \mathrm{~g} \mathrm{CaO}$.

\section{Ueber Entkälken, Schwellen, Pickeln.}

Bei diesen gerberischen Arbeiten spielt das Verhalten der Haut' gegenüber Säuren eine wichtige Rolle. V. Kubelka ${ }^{53}$ ) hat die Adsorptionserscheinungen beim Zusammenbringen von Hautpulver mit Ameisensäure, Essigsäure, Propionsäure und Buttersäure untersucht und gefunden, daß die in der Lösung stattfindenden Konzentrationsänderungen rasch einem Gleichgewichtszustand zueilen derart, dab sie schon nach fünf Sekunden 50 Proz, der gesamten Veränderungen ausmachen. Die Geschwindigkeit der Konzentrationsänderung nimmt mit steigender Konzentration der Säure zu. Die quantitativen Verhältnisse beim Gleichgewicht lassen sich befriedigend durch die Adsorptionsformel ausdrücken.

s) D. Mc. Candlish und A. Wilson, Coll. 1913. 85.

52) J. He $1 \mathrm{frich}$, Journ. of The American Leather Chemists Association 1915, 397.

53) V. Kubelka, Collegium 1915, 389.
H. Procte ${ }^{54}$ ) hat gefunden, dab bei der Schwellung der Gelatine in verdünnten Säuren ein ionisiertes Gelatinesalz gebildet wird und daß die Schwellung der Gelatine von dem osmotischen Druck und dem Gleichgewichtszustande zwischen dem Gelatinesalz und der außerhalb der Gelatine befindlichen Säure abhängt. Wenn die Ionisationskonstanten und das Molekulargewicht des Gelatinesalzes bekannt sind, können alle anderen bei der Schwellung in Betracht kommenden Größen bestimmt werden.

G. Pow ar nin ${ }^{55}$ ) hat auf Grund der früheren eingehenden Arbeiten von J. Paes s ler und W. Appelius ${ }^{56}$ ) über das Verhalten der BlöBe gegenüber Säuren eine Formel zur Bestimmung der Blößenquellung unter dem Einfluß von Säuren abgeleitet.

H. Procter ${ }^{57}$ ) erörtert die auf Grund wissenschaftlicher Forschungen sich ergebenden Grundsätze für das praktische Entkälken mit Säuren. Eine vollständige Entkälkung kann nur erreicht werden, wenn man eine stark verdünnte Säurelösung verwendet. Es darf auch nur wenig mehr von dieser verdünnten Säure verwendet werden, als zur Bindung des Kalkes erforderlich ist, da sonst ein Tèil der überschüssigen Säure von der Haut gebunden wird und auf diese schädlich einwirkt. Die Wirkung der Säure kann durch Zugabe eines neutralen Salzes wesentlich abgeschwächt werden. Versetzt man z. B. eine Lösung von Essigsäure mit der gleichen Menge von essigsaurem Natrium, so wird die Schwellwirkung auf $1 / 80$ derjenigen der Essigsäure herabgesetzt. In ähnlicher Weise wirken bei Verwendung von organischen Säuren die durch Einwirkung auf Kalk entstehenden Jöslichen Kalksalze dieser Säuren, so daß man frische Säurelösungen zur Abschwächung der Säurewirkung nur mit einer schon Kalksalze enthaltenden alten Entkälkungsflüssigkeit zu versetzen braucht. Es ist auch geraten worden, die schwachen Säuren, durch die billigeren starken Säuren aus dem Kalksalz zu befreien und dadurch wieder verwendbar zu machen. Es ist dabei jedoch mit dem Einflub der dabei gebildeten Neutralsalze der starken Säuren und sodann auch mit der vom Autor festgestellten Tatsache zu rechnen, daß bei gleichzeitigem Vorhandensein von Neutralsalzen starker Säuren sowie von schwachen Säuren ein Teil der starken

j+) H. Procter, Collegium 1914, 194.

55) G. Powarnin, Collegium 1914, 659.

56) J. Paessler und W. Appelius, Deutsche Gerberzeitung 1902, Nr. 47 und Collegium 1902, 179.

${ }^{57)}$ H. Procter, Journ. of The American Leather Chemists Association 1913, 350. 
Säure in Freiheit gesetzt wird. Am empfehlenswertesten erscheint die Verwendung einer starken Säure, deren Kalksalz wenig löslich ist, was besonders bei Schwefelsäure zutrifft.

Die neueren patentierten Entkälkungsverfahren suchen auf verschiedenem Wege meist auch das Ziel zu erreichen, daß das Auftreten schädlicher. Mengen überschüssiger Säure oder die mit überschüssiger Säure verbundene schädliche Wirkung auf die Haut vermieden wird. $\mathrm{Zu}$ diesem Zwecke sind von H. Procter ${ }^{58}$ ) schon früher saures schwefelsaures Natrium und saures phosphorsaures Natrium vorgeschlagen worden. Auch bei einem neuen Entkälkungsverfahren von P. Schneidèr (D. R.P. Nr. 268 236) ${ }^{59}$ ) werden saure Salze unter Zusatz von überschüssigem Kochsalz sowie von Borsäure und Zucker oder zuckerhaltigen Stoffen verwendet. Später hat Sch neider das Verfahren in der

$\left.{ }^{58}\right)$ H. Procter, The Prinziples of Leather Manufacture 1913,156

$\left.{ }^{59}\right)$ Patentbeschr. s. Collegium 1914, 48.
Weise ausgebildet (D.R.P. Nr. 268994) ${ }^{60}$ ), daß an Stelle der sauren Salze zwei oder mehr Säuren oder Salze solcher Säuren gleichzeitig verwendet werden, von denen die eine zur Gruppe der stärkeren, die andere zur Gruppe der schwächeren Säuren gehört und außerdem eine an eine flüchtige Base gebundene Säure (z. B. Ammoniumformiat oder das billigere Chlorammonium) oder Zucker zugesetzt wird.

G. Grasse ${ }^{61}$ ) erörtert die theoretisch möglichen Vorgänge in dem am häufigsten angewandten Kochsalz-Schwefelsäurepickel mit einem Gehalt von 8--10 Proz. Kochsalz und $1-2$ Proz. konzentrierter Schwefelsäure. Im fertig gebildeten Pickel sind nur $\mathrm{NaCl}, \mathrm{HCl}$ und $\mathrm{Na}_{2} \mathrm{SO}_{4}$ vorhanden. Es genügt daher zur technischen Kontrolle der Zusammensetzung des Pickels eine Bestimmung des Gehaltes an freier Salzsäure sowie an Kochsalz.

(Fortsetzung folgt.)

$\left.{ }^{80}\right)$ Patentbeschr. s. Collegium 1914, 86.

${ }^{61)}$ C. Grasser, „Technikum" (Beilage des „Ledermarkt") 1913, 105.

\section{Kolloidchemie und Metallurgie. ${ }^{*}$}

Von Wolfgang Ostwald (Leipzig).

Ich komme nun $z u$ einem besonders wichtigen technischen Anwendungsgebiet der Kolloidchemie, den Anwendungen in der Metallurgie. Allerdings, muß ich vorausschicken, daB die Möglichkeiten, welche die Kolloidchemie für die Erklärung zahilreicher metallurgischer Probleme bietet, heute noch ganz und gar nicht ausgenutzt worden sind. Und wenn ich im folgenden mit etlaube, Ihnen einige eigene kolloidchemische Gesichtspunkte zur Beurteilung metallurgischer Fragen zu geben, die Sie beim Studium der einschlägigen Literatur nicht finden würden, so wage ich dies nur in der festen Ueberzeugung, daß die kolloidchemische resp. dispersoidchemische Betrachtungsweise eine ganz außergewöhnliche Zukunft in der Metallurgie haben wird.

Einige bekanntere Probleme kolloidchemischer Natur finden Sie schon bei der Metall ge w in nung resp. der Erzaufbereitung. So ist es z. B. bekannt, daß das Gold ton- oder lehmartiger Erdschichten - nur außerordentlich schwierig extrahiert werden kann. Das Metall ist in diesen Materialien jedenfalls in sehr hochdisperser Form vorhanden, insbesondere aber durch die gallertartigen Hydroxyde und Silikate des Aluminiums und Eisens so fest gebunden oder umschlossen, daß die gewöhnlichen Auslaugeverfahren ungenügend sind. Die genannten

s) Vorliegende Seiten wurden einem Vortrag entnommen, der mit anderen in dem Buche ..Die Welt der vernachlässigten Dimensionen" (Verlag Th. Steinkopft, Dresden) abgedruckt ist. Die Erwigung cla in obion Darlegtingen auch Darleg hinausgehen - fermerhin aber die offensichtliche Wichtigkeit, welche geräde die Metallurgie als ein neues Anwendungsgebiet der Kolloidchemie bednspruchen dürte, falls diese neuen Gesichtspunkte richtig sein sollten, haben den Verf, zu obigem "Autoreferat" reranlaßt. hydratisierten Kolloide "maskieren" gleichsam das Gold, ähnlich wie etwa Eisen in Gegenwart resp. in sorptiver Verbindung mit manchen organischen Substanzen „maskiert" erscheint, d. h. die gewöhnlichen analytischen Reaktionen nicht oder erst nach Zerstörung des organischen Anteils ergibt. Die Nutzbarmachung solcher Erze wïrde auf eine entsprechende Zerstörung der genannten anorganischen Kolloide resp. auf eine Trennung dieser Sorptionskomplexe herauskommen, ein kolloidchemisches Problem, das anscheinend noch nicht zur Zufriedenheit gelost ist. Uebrigens wird auch relativ reines kolloides Gold z. B. beim Schütteln mit Quecksilber nicht aufgenommen, eine Tatsache, die wohl ebenfalls auf die Schwierigkeiten einer innigen Berührung der kolloiden Teilchen mit der Quecksilberoberflä̉che zurückzuführen ist.

Andere kolloidchemische Erscheinungen in der Metallurgie finden Sie bei der elektrolytischen Metallabscheidung. Hier ist bekannt, daß die Struktur des elektrolytischen Metalniederschlags weitgehend durch den Zusatz minimaler Mengen organischer Kolloide wie Gelatine, EiweiB, Dextrin usw. beeinflußt wird. In bestimmten Konzentrationen wirken diese Kolloide im Sinne einer starken Erhöhung des Dispersitätsgrades des elektrolytischen Niederschlags. Statt voluminöser makro- oder mikrokristallinischer Niederschläge entstehen außerordentlich dichte, fein strukturierte Schichten, die einen einheitlichen Glanz zeigen. Man nennt dieses Verfahren daher auch "Glanzgalvan is at $\mathrm{i}$ o $\mathrm{n}^{\text {(1) }}$ ), und

1) Siehe z. B. E. Mülier, Zeitschr. f. Elektrochem. 1906, 317 ; ausführl, Referat in Koll.-Zeitschr. 1, 60 (1906). 